\title{
Usefulness and Limitation of Dobutamine Stress Echocardiography to Predict Acute Response to Cardiac Resynchronization Therapy
}

\author{
Mario Sénéchal, M.D., F.R.C.P.C., ${ }^{*}$ Patrizio Lancellotti, M.D., Ph.D., † Patrick Garceau, M.D., \\ F.R.C.P.C., ${ }^{*}$ Jean Champagne, M.D., F.R.C.P.C., ${ }^{*}$ Michelle Dubois, B.Sc., ${ }^{*}$ Julien Magne, Ph.D., ${ }^{*}$ \\ Louis Blier, M.D., F.R.C.P.C., ${ }^{*}$ Frank Molin, M.D., F.R.C.P.C. ${ }^{*}$ François Philippon, M.D., \\ F.R.C.P.C., ${ }^{*}$ Jean G. Dumesnil, M.D., F.R.C.P.C., ${ }^{*}$ Luc Pierard, M.D., Ph.D., F.E.S.C.†, \\ and Gilles O'Hara, M.D., F.R.C.P.C.*
}

*Department of Cardiology, Institut de Cardiologie de Québec, Hôpital Laval, Québec, Canada; and $\nmid$ Department of Cardiology, CHU de Liège, University Hospital, Sart Tilman, Liège, Belgium

Background: It has been hypothesized that a long-term response to cardiac resynchronization therapy (CRT) could correlate with myocardial viability in patients with left ventricular (LV) dysfunction. Contractile reserve and viability in the region of the pacing lead have not been investigated in regard to acute response after CRT. Methods: Fifty-one consecutive patients with advanced heart failure, $L V$ ejection fraction $\leq 35 \%, Q R S$ duration $>120 \mathrm{~ms}$, and intraventricular asynchronism $\geq 50$ ms were prospectively included. The week before CRT implantation, the presence of viability was evaluated using dobutamine stress echocardiography. Acute responders were defined as a $\geq 15 \%$ increase in $L V$ stroke volume. Results: The average of viable segments was $5.8 \pm 1.9$ in responders and $3.9 \pm 3$ in nonresponders $(P=0.03)$. Viability in the region of the pacing lead had an excellent sensitivity (96\%), but a low specificity (56\%) to predict acute response to CRT. Mitral regurgitation (MR) was reduced in 21 patients (84\%) with acute response. The presence of MR was a poor predictor of response (sensibility $93 \%$ and specificity 17\%). However, combining the presence of MR and viability in the region of the pacing lead yields a sensibility (89\%) and a specificity (70\%) to predict acute response to CRT. Conclusion: Myocardial viability is an important factor influencing acute hemodynamic response to CRT. In acute responders, significant $M R$ reduction is frequent. The combined presence of $M R$ and viability in the region of the pacing lead predicts acute response to CRT with the best accuracy. (ECHOCARDIOGRAPHY, Volume **, ************)

dobutamine stress echocardiography, resynchronization therapy, ventricular dyssynchrony, heart failure, myocardial viability, mitral regurgitation

Cardiac resynchronization therapy (CRT) improves ventricular dyssynchrony, and in term is associated with an improvement in symptoms and prognosis in patients with severe heart failure. ${ }^{1-6}$ Echocardiographic assessment of the acute hemodynamic response to CRT

Dr Mario Sénéchal is a recipient of a Grant from Institut de Cardiologie de Québec

Address for correspondence and reprint requests: Mario Sénéchal, M.D., F.R.C.P.C., Institut Universitaire de Cardiologie et de Pneumologie de Québec, 2725, Chemin SainteFoy, Québec, Québec G1V 4G5, Canada. Fax: 418-656-4581; E-mail: mario.senechal@crhl.ulaval.ca predicts long-term clinical outcome in both ischemic and nonischemic cardiomyopathy. ${ }^{7}$ After CRT, about $50 \%$ of patients have an acute increase in stroke volume $\geq 15 \%$ and are identified as acute responders. ${ }^{8}$ An acute increase in stroke volume is related to reduction of left ventricle (LV) dyssynchrony and corresponding stress-strain disparities and inefficient contraction of the ventricle. ${ }^{9}$ Resynchronization of the LV improved coordinated timing of the mechanical activation of papillary muscles and appears to be the main mechanistic contributor to immediate $\mathrm{MR}$ reduction and increase in stroke volume. ${ }^{10-17}$ Response to CRT might 
be modulated by the presence of functional mitral regurgitation before implantation. In the CARE-HF study, it was shown that patients whose conditions did not improve were likely to have no significant mitral regurgitation as compared with responders. ${ }^{18}$ However, the presence of MR seems not to predict acute response to $\mathrm{CRT}^{8}$ To date, the main approach identifying CRT candidates has been QRS duration and mechanical dyssynchrony. ${ }^{19-21}$ However, between $30 \%$ and $40 \%$ of patients with congestive heart failure and QRS $>120 \mathrm{~ms}$ do not clinically improve after CRT. ${ }^{6,22}$ Moreover, even in patients with QRS $>120 \mathrm{~ms}$ and significant intraventricular asynchronism response to CRT may not occur, ${ }^{23}$ CRT nonresponse is likely a diverse phenomenon. Contractile reserve may represent a key element in the resynchronization process. Because electrical conduction and regional wall thickening are influenced by the extent of myocardial fibrosis, it has been hypothesized that a long-term response to CRT could correlate with myocardial viability in patients with $\mathrm{LV}$ dysfunction. Using nuclear ${ }^{5,6}$ myocardial perfusion imaging $(2 \mathrm{C} 1 \mathrm{Ti}),{ }^{24,25}$ magnetic resonance imaging (MRI), ${ }^{23,26}$ or dobutamine stress echocardiography (DSE), ${ }^{27-31}$ studies have demonstrated the importance of $L V$ viability in predicting response to CRT. Furthermore, scar tissue in the LV pacing lead region may prohibit response to CRT. ${ }^{32}$ However, contractile reserve and viability in the region of the pacing lead have not been investigated in regard to acute response after CRT. We therefore hypothesized that the combined presence of viability in the region of the pacing lead and MR is the best echocardiographic parameter to predict acute response following CRT.

\section{Methods}

From May 2005 to March 2008, 51 patients (mean age $66 \pm 11$ years, $35(67 \%)$ male) provided informed consent and were prospectively enrolled. Inclusion criteria were as follows: (1) NYHA functional classes III and IV heart failure, (2) QRS duration $\geq 120 \mathrm{~ms}$, (3) chronic $\mathrm{LV}$ systolic dysfunction (LV ejection fraction $\leq 35 \%$ ), (4) basal LV dyssynchrony $\geq 50 \mathrm{~ms}$, (5) optimal medical treatment for heart failure including angiotensin-converting enzyme inhibitors or AT1 receptor antagonists diuretics, beta-receptor blockers and spironolactone when tolerated, and (6) sinus rhythm. Patients with recent myocardial infarction, with coronary revascularization ( $<6$ months), and presenting standard contraindications to DSE were excluded. All patients underwent coronary angiograms before implantation to exclude treatable ischemic heart disease. Etiology was considered ischemic in the presence of significant coronary artery disease $(\geq 50 \%$ stenosis in one or more of the major epicardial coronary arteries) and/or a history of myocardial infarction or prior revascularization. All patients provided informed consent, and the study protocol was approved by local ethics committee.

\section{Study Design}

The patients underwent a clinical examination, a 12-lead electrocardiography (ECG), and a resting and DSE, within the week before CRT implantation. Resting echocardiography was also performed within 24 hours following device placement. Acute responders to CRT were defined as a $\geq 15 \%$ increase in $\mathrm{LV}$ stroke volume. $^{8}$

\section{Echocardiographic Assessment}

Echocardiographic measurements were performed by two observers blinded to patients' status using Philips Sonos 5500 or 7500 instrument with a 2.5-MHz transducer (Philips Medical Systems, Amsterdam, The Netherlands). $\mathrm{LV}$ volumes and ejection fraction were measured using the modified biplane Simpson's rule. LV stroke volume was calculated by multiplying the LV outflow tract area by the LV outflow tract velocity-time integral measured by pulsed-wave Doppler.

The proximal isovelocity surface area (PISA) was used to assess the MR severity and to measure the effective regurgitant orifice (ERO) area and regurgitant volume. ${ }^{33}$ Aortic and pulmonary Doppler flows were recorded in the pulsed mode from the apical four-chamber view and parasternal short-axis view, respectively. Aortic and pulmonary ejection delays were defined as the delay between the onset of the QRS complex on the surface ECG and the onset of the aortic and pulmonary waves. The interventricular delay was defined as the time difference between the aortic and pulmonary electromechanical delay. ${ }^{34}$

\section{Intraventricular Asynchronism Measurement}

Tissue Doppler imaging (TDI) was performed in the pulsed-wave Doppler mode from apical 
views to assess longitudinal myocardial regional function, analyzing the septal, inferior, lateral, anterior, and posterior walls. ${ }^{34}$ Velocity profiles were recorded with a sample volume placed in the middle of the basal segment of each wall. Gain and filters were adjusted as needed to eliminate background noise and to allow for a clear tissue signal. TDI signals were recorded at a sweep of $100 \mathrm{~mm} / \mathrm{s}$. The electromechanical delay defined as the delay between the onset of the QRS complex on the surface ECG and the onset of the systolic TDI wave was measured by MS or PG. Intraventricular asynchronism was defined as the time difference between the shortest and longest electromechanical delay among the five LV walls. ${ }^{34}$

\section{Assessment of Contractile Reserve}

All patients underwent DSE according to a low-dose infusion protocol. The patients received $5,10,15$, and $20 \mu \mathrm{g} / \mathrm{kg}$ per minute of dobutamine in a 3-minute stage, with echocardiographic images recorded at each stage. ${ }^{35,36}$ Heart rate and blood pressure were monitored during each stage. Criteria for stopping the dobutamine infusion included (1) hypotension (systolic blood pressure $<90 \mathrm{mmHg}$ ), (2) angina, (3) significant arrhythmias (atrial fibrillation, bigeminy, ventricular tachycardia), and (4) attainment of $85 \%$ maximal predicted heart rate. The regional wall motion was assessed by the 16-segment model recommended by the American Society of Echocardiography. ${ }^{37}$ Thus, a normal or hyperkinetic segment was graded as 1, hypokinetic as 2, akinetic as 3, and dyskinetic as 4 . The stress images at the dobutamine dose showing the maximum augmentation of wall motion were compared with baseline images. A segment was considered to have contractile reserve if after dobutamine the wall motion improved by one grade. Viability in the region of the LV pacing lead was defined as the presence of viability in two contiguous segments. DSE was interpreted by MS or PG.

\section{CRT Implantation and LV Lead Position}

A coronary sinus venogram was obtained using balloon catheter, followed by the insertion of the LV pacing lead (Guidant Corporation, St Paul, NM or Medtronic Inc., Minneapolis, MN, USA) in the coronary sinus. The preferred position was a lateral or posterolateral vein. The right atrial and ventricular leads were positioned conventionally. All leads were con- nected to a dual-chamber biventricular pacing (Guidant Corporation, or Medtronic Inc., Milwaukee, WI, USA). One day after implantation, the LV lead position was assessed from a chest x-ray. Using the frontal and lateral views (scored anterior, lateral, or posterior), we determined the LV lead locations. ${ }^{38}$

\section{Statistical Analysis}

Results are expressed as mean \pm SD or percentages unless otherwise specified. The patients were separated into two groups (responders and nonresponders) according to the early post-CRT change in LV stroke volume (>15\%). ${ }^{8}$ Interobserver and intraobserver variabilities for the measurement of inter- and intraventricular asynchronism as well as for the quantification of the wall motion score index (WMSI) were determined from the analysis of Doppler echocardiographic images of 15 randomly selected patients by two independent observers (MS and PG). The results were compared with a one-way analysis of variance, Pearson's correlation coefficient, and the Bland-Altman method. Baseline data of the responder group versus the nonresponder group were compared for statistical significance using the $t$-test or chi-square test, as appropriate. Baseline and post-CRT MR severity were compared within groups using the paired- $t$ test or chi-square test, as appropriate. Sensitivity and specificity for prediction of CRT response were determined for various cutoff values of the echocardiographic parameters using receiver-operating characteristic curves. Linear regression analyses were used to evaluate the relationship between CRT response, assessed as the percentage of change in LV stroke volume, and the percentage of change in echocardiographic data.

\section{Results}

\section{Patients}

The day after CRT implantation, 28 patients (55\%) were responders and compared to nonresponders $(\mathrm{n}=23,45 \%)$ there was no significant difference with regards to baseline demographic and clinical characteristics (Table I). However, the patients in the nonresponder group tended to have higher LV stroke volume ( $46 \pm 2 \mathrm{ml}$ vs. $39 \pm 12 \mathrm{ml}, \mathrm{P}=0.06)$. The number of akinetic segments in each patient ranged from 1 to 15 segments (mean 9.5 \pm 3.3). Device implantation was successful in all patients and one patient developed pneumothorax after 
SÉNÉCHAL, ET AL.

TABLE I

Demographic and Clinical Data

\begin{tabular}{|c|c|c|c|c|}
\hline Variables & $\begin{array}{l}\text { All Patients } \\
\quad(\mathrm{n}=51)\end{array}$ & $\begin{array}{l}\text { Responders } \\
(\mathrm{n}=28,55 \%)\end{array}$ & $\begin{array}{l}\text { Nonresponders } \\
(\mathrm{n}=23,45 \%)\end{array}$ & P-Value \\
\hline \multicolumn{5}{|l|}{ Demographic data } \\
\hline Age (years) & $66 \pm 11$ & $67 \pm 10$ & $65 \pm 13$ & 0.52 \\
\hline Male, n (\%) & $35(69)$ & $19(68)$ & $16(70)$ & 0.90 \\
\hline $\mathrm{CAD}, \mathrm{n}(\%)$ & $35(69)$ & $18(65)$ & $17(74)$ & 0.46 \\
\hline \multicolumn{5}{|l|}{ Clinical data } \\
\hline QRS duration (ms) & $161 \pm 30$ & $159 \pm 27$ & $163 \pm 33$ & 0.67 \\
\hline LBBB, n $(\%)$ & $32(63)$ & $20(71)$ & $12(52)$ & 0.16 \\
\hline $\mathrm{RBBB}, \mathrm{n}(\%)$ & $3(6)$ & $2(7)$ & $1(4)$ & 0.67 \\
\hline IVCD, n (\%) & $8(17)$ & $3(12)$ & $5(22)$ & 0.36 \\
\hline $\mathrm{PR}(\mathrm{ms})$ & $184 \pm 41$ & $176 \pm 32$ & $194 \pm 49$ & 0.14 \\
\hline Pre-CRT pacing, n (\%) & $8(16)$ & $3(11)$ & $5(22)$ & 0.28 \\
\hline NYHA III/IV, n (\%) & $35(69) / 16(31)$ & $21(75) / 7(25)$ & $14(60) / 9(39)$ & 0.28 \\
\hline \multicolumn{5}{|l|}{ Medication } \\
\hline Diuretic, n (\%) & $48(94)$ & $26(93)$ & $22(96)$ & 0.67 \\
\hline$\beta$-blockers, n (\%) & $48(94)$ & $26(94)$ & $22(96)$ & 0.67 \\
\hline ACEi, n (\%) & $35(69)$ & $19(68)$ & $16(70)$ & 0.90 \\
\hline AR blockers, n (\%) & $15(30)$ & $9(33)$ & $6(26)$ & 0.58 \\
\hline Digoxin, n (\%) & $14(27)$ & $5(18)$ & $9(39)$ & 0.09 \\
\hline Spironolactone, n (\%) & $33(63)$ & $15(54)$ & $17(74)$ & 0.14 \\
\hline
\end{tabular}

$\mathrm{CAD}=$ coronary arteries disease $\mathrm{LBBB}=$ left bundle branch block; RBBB $=$ right bundle branch block; IVCD $=$ intraventricular conduction defect; $\mathrm{ACEi}=$ angiotensin-converting enzyme inhibitors; $\mathrm{AR}=$ angiotensin receptors.

CRT implantation. LV pacing threshold was not different between responders and nonrespon$\operatorname{ders}(1.18 \pm 0.70$ vs. $1.75 \pm 0.5, \mathrm{P}=0.17)$. In the subgroup of patients with $\mathrm{CAD}$, no patients experienced angina, electric, or regional wall motion modification at peak stress $(20 \mu \mathrm{g} / \mathrm{kg}$ per minute) suggesting ischemia.

\section{Reproducibility of Asynchronism and WMSI}

There were excellent correlations $(r \geq 0.96)$ between intra- and interobserver analyses of viability in the region of the pacing lead and for WMSI. Intra- and interobserver relative differences were $<3 \%$ for all parameters. The Bland-Altman method showed excellent agreement between inter- and intraobserver measurements in both low and high values of asynchronism or WMSI.

\section{Contractile Reserve to Predict Response}

All patients completed the DSE protocol without complications. During low-dose dobutamine infusion, responders tended to have less akinetic segments $(7.7 \pm 3$ vs. $9 \pm 3, \mathrm{P}=0.06)$ and a significantly higher number of viable seg- ments $(5.8 \pm 1.94$ vs. $3.87 \pm 2.99, \mathrm{P}=0.007)$ than nonresponders. The presence of more than four viable segments and viability in the region of the pacing lead were statistically more frequent in responders $(96 \%$ vs. $52 \%, \mathrm{P}<0.0001$ and $96 \%$ vs. $43 \%, \mathrm{P}<0.0001$, respectively) (Table II). LV stroke volume changes after CRT were directly related to the improvement in WMSI during dobutamine infusion $(\mathrm{r}=0.45$, $\mathrm{P}=0.0012$ ) (Fig. 1A). A similar correlation was also observed between the change in ERO after CRT and the improvement of WMSI during $\operatorname{DSE}(\mathrm{r}=0.41, \mathrm{P}=0.0057)$ (Fig. 1B).

\section{Global Viability and Local Viability versus Response to CRT}

Among patients with local viability (i.e., viability in the region of the pacing lead), 27 (73\%) were responders corresponding to $96 \%$ of all responders. Conversely, in patients with global viability (i.e., $\geq 4$ viable segments) without local viability $(\mathrm{n}=4)$, only one patient $(25 \%)$ was identified as a responder. In the absence of local and global viabilities, all patients $(\mathrm{n}=10$, $100 \%$ ) were nonresponders (Fig. 2). 
TABLE II

Echocardiographic Data

\begin{tabular}{|c|c|c|c|c|}
\hline Variables & $\begin{array}{l}\text { All Patients } \\
\quad(\mathrm{n}=51)\end{array}$ & $\begin{array}{c}\text { Responders } \\
(\mathrm{n}=28,55 \%)\end{array}$ & $\begin{array}{l}\text { Nonresponders } \\
(\mathrm{n}=23,45 \%)\end{array}$ & P-Value \\
\hline \multicolumn{5}{|l|}{ LV geometry and function } \\
\hline LV end-diastolic volume (ml) & $214 \pm 67$ & $211 \pm 69$ & $217 \pm 65$ & 0.75 \\
\hline LV end-systolic volume (ml) & $178 \pm 63$ & $177 \pm 67$ & $180 \pm 67$ & 0.90 \\
\hline LV end-diastolic diameter (mm) & $67 \pm 8$ & $66 \pm 8$ & $68 \pm 8$ & 0.26 \\
\hline LV end-systolic diameter (mm) & $59 \pm 9$ & $61 \pm 9$ & $57 \pm 3$ & 0.13 \\
\hline End-systolic SI (\%) & $63 \pm 9$ & $64 \pm 9$ & $63 \pm 9$ & 0.87 \\
\hline End-diastolic SI (\%) & $69 \pm 9$ & $66 \pm 2$ & $65 \pm 2$ & 0.82 \\
\hline LV stroke volume (ml) & $42 \pm 12$ & $39 \pm 12$ & $46 \pm 2$ & 0.06 \\
\hline LV ejection fraction $(\%)$ & $19 \pm 7$ & $18 \pm 8$ & $19 \pm 6$ & 0.70 \\
\hline \multicolumn{5}{|l|}{ Asynchronism } \\
\hline Interventricular (ms) & $45 \pm 27$ & $43 \pm 26$ & $47 \pm 28$ & 0.63 \\
\hline Intraventricular (ms) & $83 \pm 25$ & $87 \pm 25$ & $79 \pm 24$ & 0.23 \\
\hline \multicolumn{5}{|l|}{ No. of akinetic segments } \\
\hline Rest & $9.5 \pm 3$ & $9.4 \pm 3$ & $10 \pm 3$ & 0.26 \\
\hline Dobutamine & $8.4 \pm 3$ & $7.7 \pm 3$ & $9 \pm 3$ & 0.06 \\
\hline \multicolumn{5}{|l|}{ Wall motion score index } \\
\hline Rest & $3.5 \pm 0.4$ & $3.41 \pm 0.42$ & $3.55 \pm 0.25$ & 0.15 \\
\hline Dobutamine & $3.1 \pm 0.5$ & $2.97 \pm 0.41$ & $3.25 \pm 0.48$ & 0.03 \\
\hline \multicolumn{5}{|l|}{ Viability } \\
\hline No. of viable segments & $4.9 \pm 3$ & $5.8 \pm 1.94$ & $3.87 \pm 2.99$ & 0.007 \\
\hline More than viable segments, $\mathrm{n}(\%)$ & $39(76)$ & $27(96)$ & $12(52)$ & $<0.0001$ \\
\hline Viability in the region of the lead, $\mathrm{n}(\%)$ & $37(73)$ & $27(96)$ & $10(43)$ & $<0.0001$ \\
\hline \multicolumn{5}{|l|}{ Lead placement } \\
\hline Posterior, n (\%) & $34(66)$ & $20(71)$ & $14(61)$ & 0.83 \\
\hline Lateral, $\mathrm{n}(\%)$ & $15(30)$ & $8(29)$ & $7(30)$ & 0.87 \\
\hline Anterior, $\mathrm{n}(\%)$ & $2(4)$ & - & $2(9)$ & - \\
\hline
\end{tabular}

$\mathrm{LV}=$ left ventricular; $\mathrm{ED}=$ end-diastolic $; \mathrm{ES}=$ end-systolic $; \mathrm{SI}=$ sphericity index.

\section{Impact of Viability and Mitral Regurgitation on CRT Response}

The prevalence of MR between responders and nonresponders was not statistically different before and after CRT (pre-CRT: 93\% vs. $83 \%, \mathrm{P}=0.26$, post-CRT: $83 \%$ vs. $85 \%$, $\mathrm{P}=0.96$ ). Moreover, there was no significant difference in baseline MR severity between groups (Fig. 3A), and whereas in responders ERO and regurgitant volume were significantly reduced following CRT, there was no significant change in nonresponders (Fig. $3 \mathrm{~A}$ and $\mathrm{B}$ ). Indeed, in responders, ERO was reduced by $57 \pm$ $24 \%$ (from $18 \pm 12 \mathrm{~mm}^{2}$ to $8 \pm 8 \mathrm{~mm}^{2}, \mathrm{P}=$ $<0.001)$. The percentage of patients with severe $\mathrm{MR}\left(\mathrm{ERO} \geq 20 \mathrm{~mm}^{2}\right)$ was also not statistically different between groups before CRT (Fig. 3C). Only four responders had no MR before CRT. In receiver-operating characteristics curves, the presence of MR, as well as the presence of viability on the region of the pacing lead, was associated with excellent sensitivity
(93\% and $96 \%)$ but with low specificity (17\% and $56 \%$ ) in predicting acute CRT response. Combining the presence of MR and viability in the region of the pacing lead yield the best combination of sensitivity and specificity $(89 \%, 70 \%)$ (Fig. 4).

\section{Discussion}

The main finding of the present study showed that for acute benefit in ischemic and nonischemic cardiomyopathies, CRT requires the presence of myocardial viability. A direct relationship between improvement in WMSI as assessed during low-dose dobutamine infusion and the improvement in LV stroke volume and reduction in ERO after CRT was described. This study shows the ability of DSE to predict acute response to CRT in patients with drugrefractory systolic heart dysfunction and significative intraventricular dyssynchrony.

Lastly, the combined presence of viability in the region of the pacing lead defined as viability 
A

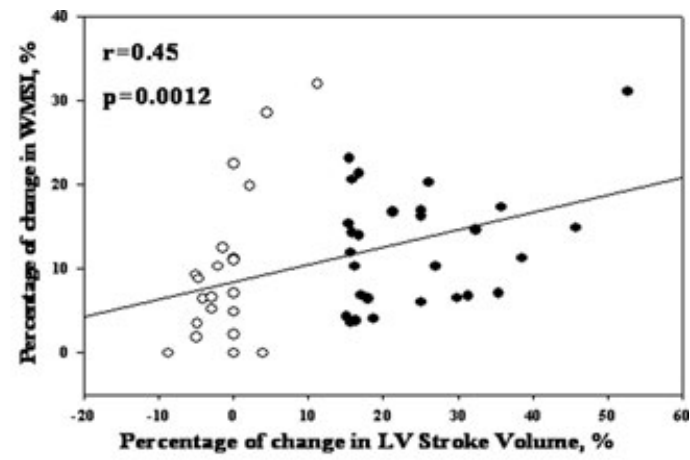

Non-responders

- Responder s

B

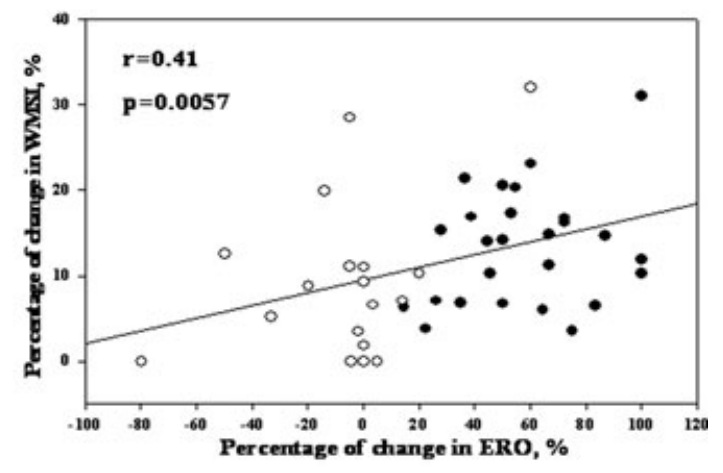

Non-responders

- Responders

Figure 1. A. Correlation between changes in WMSI (rest/dobutamine) and changes in stroke volume after CRT. B. Correlation between changes in WMSI (rest/dobutamine) and changes in ERO after CRT.

in two contiguous segments and MR predicts acute response with the best accuracy.

\section{Effect of Global Viability}

Wall motion response during dobutamine infusion is useful in predicting functional myocardial recovery in patients with ischemic and nonischemic heart diseases. ${ }^{35,39-41}$ The clinical response to treatments such as $\beta$ blockade or revascularization in patients with LV systolic dysfunction has been shown to be dependent on the presence and extent of viable myocardium. Although CRT improves cardiac function by other mechanisms than revascularization or by up-regulation of sarcoplasmic reticulum calcium ATPase (beta-blockade), the relationship between viability and CRT benefit still holds. ${ }^{27-31}$ When myocytes have been supplanted by replacement fibrosis because of cell death and interstitial remodeling, medical

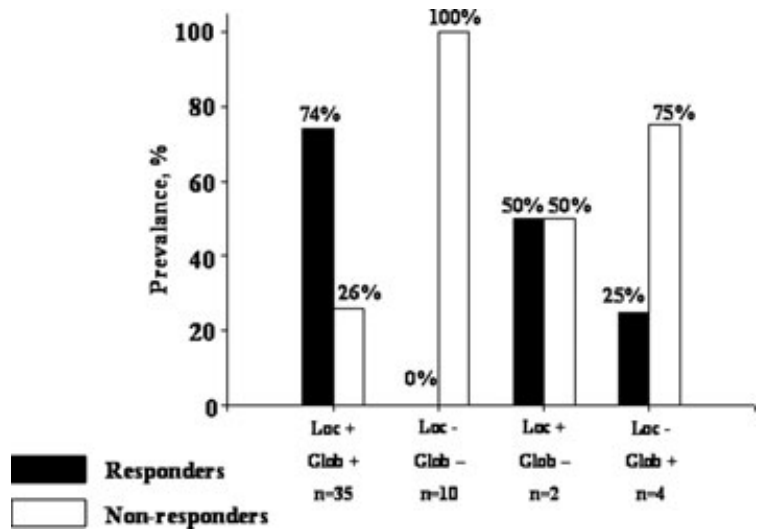

Figure 2. Percentage of responders to CRT for four different patient categories based on the presence or the absence of viability in the region of the pacing lead (local $+/$ local -) in combination with the presence or the absence of four or more viable segments (global +/global-).

therapy and CRT may not improve LV function. However, studies evaluating the relationship between CRT and myocardial viability are scarce. Ypenburg et al. evaluated and demonstrated that besides the presence of LV dyssynchrony, myocardial contractile reserve (resulting in a $\geq 7.5 \%$ increase in $\mathrm{LV}$ ejection fraction during dobutamine infusion) predicts LV reverse remodeling and improvement in $\mathrm{LV}$ function, 6 months after CRT implantation. Another study in 67 patients (34\% ischemic) revealed that the presence of contractile myocardial reserve was an independent predictor of event-free survival after CRT. Using a cutoff value of $25 \%$ increase in dobutamine $L V$ ejection fraction exhibits a sensitivity of $70 \%$ and a specificity of $62 \%$ for predicting major cardiac events. ${ }^{31}$ Hummel et al., in 21 CRT patients (100\% ischemic), evaluated myocardial viability by myocardial contrast echocardiography. ${ }^{30}$ The LV systolic performance was assessed by echocardiography on the day after implantation. In that study, acute improvement in LV stroke volume was significantly correlated with the degree of viability as determined by the perfusion score index. ${ }^{30}$ In our study, we related global viability to acute response to CRT. Improvement in LV stroke volume correlated $(\mathrm{r}=$ $0.45, \mathrm{P}=0.0012$ ) with improvement in WMSI during the dobutamine test. Moreover, responders showed a greater number of viable segments $(5.8 \pm 1.94$ vs. $3.87 \pm 2.99, \mathrm{P}=0.007)$.

The presence of more than four viable segments predicted acute response to CRT with a high sensitivity but with a low specificity. 

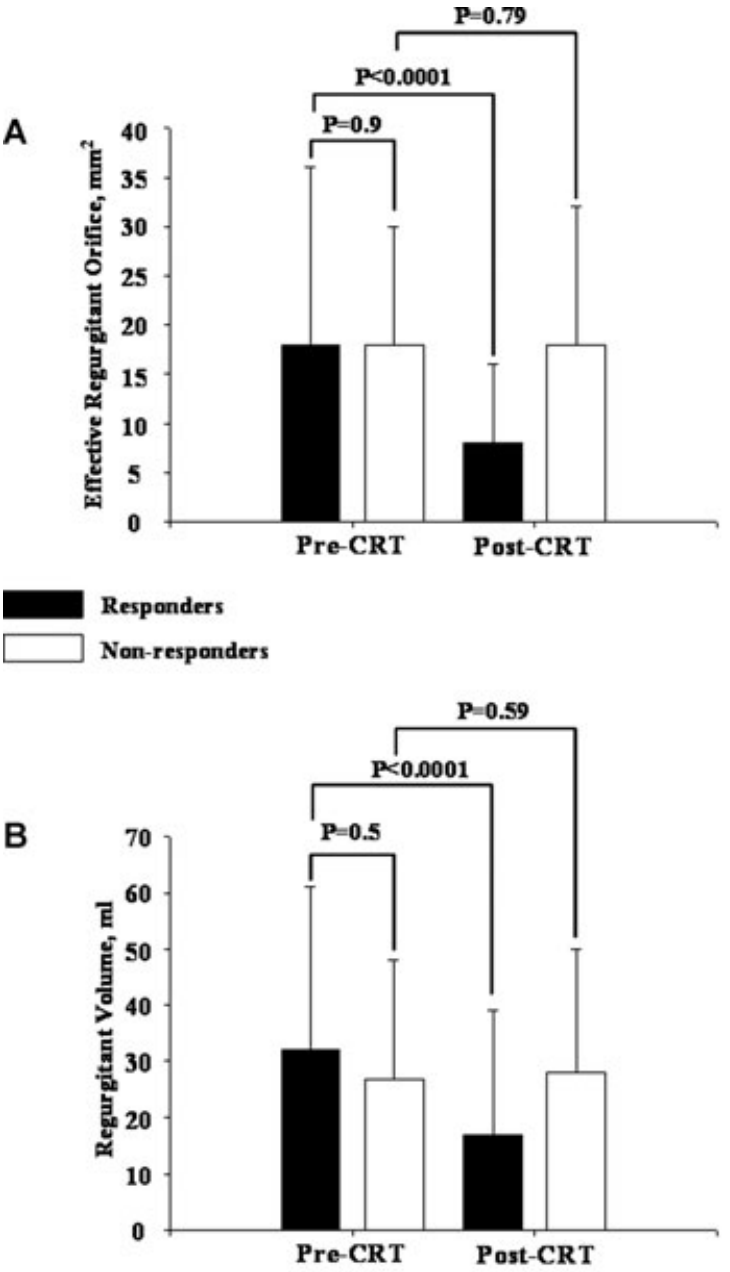

Responders

Non-responders
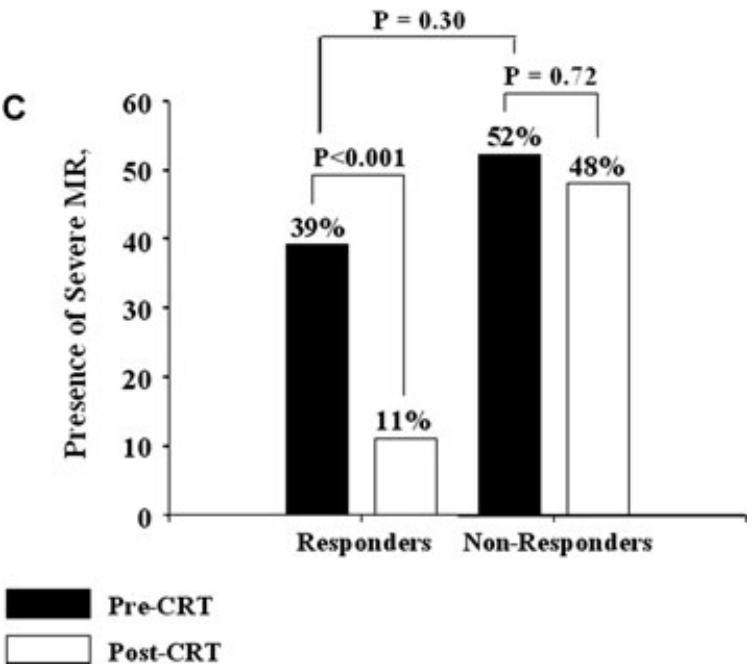

Figure 3. Quantification of $M R$ in responders and nonresponders before and after CRT. A. Changes in ERO; $\boldsymbol{B}$. Changes in regurgitant volume; $\boldsymbol{C}$. Changes in the presence of severe $M R\left(\geq 20 \mathrm{~mm}^{2}\right)$

\section{Role of Viability in the Region of the Pacing Lead}

Using contrast-enhanced MRI, Bleeker et al. demonstrated in 40 patients (100\% ischemic) that CRT did not reduce LV dyssynchrony in patients with transmural scar tissue in the posterolateral LV segments, resulting in clinical and echocardiographic nonresponse to CRT. Only $14 \%$ of patients with a posterolateral scar showed response to CRT. Even in the subset of patients with intraventricular asynchrony and postero-lateral scar, the response rate was low $(\mathrm{n}=2,18 \%){ }^{32}$ Ypenburg et al. recently observed in $31 \mathrm{CRT}$ patients that responders showed an increase in strain in the region of the LV pacing lead during low-dose dobutamine infusion while nonresponders had no contractile reserve (absence of an increase in strain). ${ }^{27}$ Furthermore, Lim et al. demonstrated that only patients $(n=19)$ with contractile reserve in the LV target site for pacing (lateral, posterolateral) presented a decrease in LV dyssynchrony with CRT. ${ }^{28}$ The authors also showed that the mean increase of LV stroke volume was greater in patients with contractile reserve $(22 \%$ vs. $0 \%)$. The number of viable segments in each wall showing viability (i.e., contractile reserve) was however not stated in that study. In line with these results, the present study demonstrated that acute responders to CRT showed viability in the region of the pacing lead

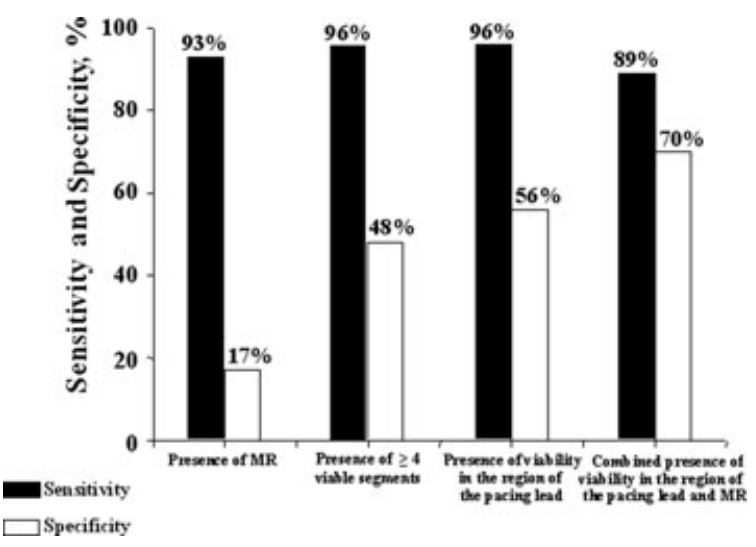

Figure 4. Performance of viability and MR evaluated before CRT in predicting acute response. 
significantly more often than nonresponders. It appears likely that viability of the paced segments is the crucial factor mediating the influence of viability (local viability vs. global) on response to CRT. Of interest, in the present study, in the absence of viability in the region of the pacing lead, three patients $(75 \%)$ did not show acute response even if they had more than four viable segments. Moreover, one patient (50\%) was a responder in the presence of only three viable segments (i.e., only in the region of the pacing lead). Therefore, it is likely that acute improvement in LV stroke volume after biventricular pacing is driven mainly by viability of the paced myocardial segments. However, viability in the region of the pacing lead predicts acute response with an excellent sensitivity $(96 \%)$ but with a low specificity (56\%). Combining viability in the region of the pacing lead and MR predicts acute response after CRT with the best accuracy suggesting that reduction of $\mathrm{MR}$ is almost mandatory in acute responders. The principal mechanism explaining this acute change in LV stroke volume is probably, at least in part, related to the diminution of functional MR. CRT appears to increase mitral closing force, coordinate tethering forces on papillary muscles, and increase the leaflet coaptation surface to reduce MR. ${ }^{13-15}$ Previous studies have reported conflicting results regarding the presence and severity of MR and response to CRT. Of interest, none of those studies have evaluated $L V$ viability and its relationship with MR presence or severity and LV remodeling. ${ }^{42-45}$ In our study, only four responders did not have MR in pre-CRT. Of interest, those patients had nonischemic cardiomyopathy, viability in the region of the pacing lead, and a mean of 8 viable segments, which suggests that acute response may occur in patients without MR before CRT but only in the presence of substantial viable myocardium.

\section{Clinical Implications}

Our present results confirm earlier suggestions that the absence of viability in the region of the LV pacing lead may prohibit response to CRT. This study focuses on acute response and its relationship with viability and MR. Even if acute response after CRT underestimates long-term effects, identification of such patients may be important since acute hemodynamic response to CRT predicts long-term clinical outcome and acute responders may represent more than $70 \%$ of all eventual responders. ${ }^{8}$ Our re- sults support that the presence of viability in the region of the pacing lead is a better predictor of acute response over the burden of global viability. Also, in the presence of local viability, a decrease of MR seems mandatory in most patients with acute response. Of interest, in our study, the criterion used to define the presence of "significant" viability in the region of the LV pacing lead (presence of viability in two contiguous segments) is simple, rapid, and easily applicable in the context of clinical evaluation before CRT. This underlines the importance of assessing local viability in order to guide $\mathrm{LV}$ positioning. The region of myocardium without viability should be avoided as a final resting place for LV lead placement to maximize the possibility of therapeutic benefit.

\section{Study Limitations}

These results should be regarded cautiously, and some limitations should be underlined. First, the lack of difference between responders and nonresponders regarding the presence of $\mathrm{CAD}$ may be the result of the small number of patients, and results should be confirmed by a larger study. Second, although the difference was not statistically different, more nonresponders took digoxin and spironolactone than responders and showed a higher incidence of class IV NYHA; therefore, because of the sample size $(\mathrm{n}=51)$ and the heterogeneity of the population studied, those data should be interpreted cautiously until confirmed by suitably powered clinical trials that are undoubtedly needed. Third, dyssynchrony was defined by longitudinal tissue Doppler imaging using a cutoff value of $50 \mathrm{~ms}$ as inclusion criterion. Combining longitudinal and radial dyssynchrony indices as inclusion criteria could have been helpful in choosing a more homogenous population prone to $\mathrm{CRT}$ response. ${ }^{46}$

\section{Conclusion}

In this study, we demonstrated that myocardial viability is an important factor influencing acute hemodynamic response to CRT. In acute responders, significant $\mathrm{MR}$ reduction is frequent. The combined presence of MR and viability in the region of the pacing lead defined as two contiguous viable segments, determined by DSE predicts acute response to CRT with the best accuracy. 


\section{DSE PREDICTS ACUTE CRT RESPONSE}

\section{References}

1. Cazeau S, Leclercq C, Lavergne T, et al: Effects of multisite biventricular pacing in patients with heart failure and intraventricular conduction delay. $N \mathrm{Engl}$ J Med 2001;344:873-880.

2. Auricchio A, Stellbrink C, Sack S, et al: Pacing Therapies I Congestive Heart Failure (PATH-CHF) Study Group: Long-term clinical effect of hemodynamically optimized cardiac resynchronization therapy in patients with heart failure and ventricular conduction delay. J Am Coll Cardiol 2002;39:2026-2033.

3. Higgins SL, Hummel JD, Niazi IK, et al: Cardiac resynchronization therapy for the treatment of heart failure in patients with intraventricular conduction delay and malignant ventricular arrhythmias. $J \mathrm{Am}$ Coll Cardiol 2003;42:1454-1459.

4. Abraham WT, Fisher WG, Smith AL, et al. MIRACLE Study Group: Multicenter InSync randomized clinical evaluation. Cardiac resynchronization in chronic heart failure. N Engl J Med 2002;346:1845-1853.

5. Bristow MR, Saxon LA, Boehmer J, et al. Comparison of Medical Therapy, Pacing and Defibrillation in Heart Failure (COMPANION) Investigators: Cardiac resynchronization therapy with or without an implantable defibrillator in advanced chronic heart failure. $N \mathrm{Engl}$ $J$ Med 2004;350:2140-2150.

6. Cleland JG, Daubert JC, Erdmann E, et al: The effect of cardiac resynchronization on morbidity and mortality in heart failure. $N$ Engl J Med 2005;352:15391549.

7. Tournoux FB, Alabiad C, Fan D, et al: Echocardiographic measure of acute haemodynamic response after cardiac resynchronization therapy predicts long term clinical outcome. Eur Heart J 2007;28:11431148.

8. Gorscan III J, Kanzaki H, Bazaz R, et al: Usefulness of echocardiographic tissue synchronization imaging to predict acute response to cardiac resynchronization therapy. Am J Cardiol 2004;93:1178-1181.

9. Bleeker GB, Mollema SA, Holman ER, et al: Left ventricular resynchronization is mandatory for response to cardiac resynchronization therapy: Analysis in patients with echocardiographic evidence of left ventricular dyssynchrony at baseline. Circulation 2007;116:1440-1448.

10. Nof E, Glikson M, Bar-Lev D, et al: Mechanism of diastolic mitral regurgitation in candidates for cardiac resynchronization therapy. Am J Cardiol 2006;97:1611-1614.

11. Ypenburg C, Lancellotti P, Tops LF, et al: Mechanism of improvement in mitral regurgitation after cardiac resynchronization therapy. Eur Heart $J$ 2008;29:757765 .

12. Breithardt OA, Sinha AM, Schwammenthal E, et al: Acute effects of cardiac resynchronization therapy on functional mitral regurgitation in advanced systolic heart failure. J Am Coll Cardiol 2003;41:765770 .

13. Kanzaki H, Bazaz R, Schwartzman D, et al: A mechanism for immediate reduction in mitral regurgitation after cardiac resynchronization therapy. $J$ Am Coll Cardiol 2004;44:1619-1625.

14. Vinereanu D, Turner MS, Bleasdale RA, et al: Mechanisms of reduction of mitral regurgitation by cardiac resynchronization therapy. $J$ Am Soc Echocardiogr 2007;20:54-62.

15. Karvounis HI, Dalamaga EG, Papadopoulos CE, et al:
Improved papillary muscle function attenuates functional mitral regurgitation in patients with dilated cardiomyopathy after cardiac resynchronization therapy. J Am Soc Echocardiogr 2006;19:1150-1157.

16. Agricola E, Oppizzi M, Galderisi M, et al: Role of regional mechanical dyssynchrony as a determinant of functional mitral regurgitation in patients with left ventricular systolic dysfunction. Heart 2006;92:1390 1395.

17. Madaric J, Vanderheyden M, Van Laethem C, et al: Early and late effects of cardiac resynchronization therapy on exercise-induced mitral regurgitation: Relationship with left ventricular dyssynchrony, remodeling and cardiopulmonary performance. Eur Heart $J$ 2007;28:2134-2141.

18. Diaz-Infante E, Mont L, Leal J, et al: Predictors of lack of response to resynchronization therapy. Am J Cardiol 2005;95:1436-1440.

19. Nelson GS, Curry CW, Wyman BT, et al: Predictors of systolic augmentation from left ventricular preexcitation in patients with dilated cardiomyopathy and intraventricular conduction delay. Circulation 2000;101:2703-2709.

20. Auricchio A, Stellbrink C, Butter C, et al. Pacing therapies in Congestive Heart Failure II Study Group, Guidant Heart Failure Research Group: Clinical efficacy of cardiac resynchronization therapy using left ventricular pacing in heart failure patients stratified by severity of ventricular conduction delay. $\mathrm{J} \mathrm{Am} \mathrm{Coll}$ Cardiol 2003;42:2109-2116.

21. Kass DA: Predicting cardiac resynchronization response by QRS duration: The long and short of it. $J$ Am Coll Cardiol 2003;42:2125-2127.

22. Leclercq C, Kass DA: Retiming the failing heart: Principles and current status of cardiac resynchronization. $J$ Am Coll Cardiol 2002;39:194-201.

23. White JA, Yee R, Yuan X, et al: Delayed enhancement magnetic resonance imaging predicts response to cardiac resynchronization therapy in patients with intraventricular dyssynchrony. $J$ Am Coll Cardiol 2006;48:1953-1960.

24. Ypenburg C, Schalij MJ, Bleeker GB, et al: Extent of viability to predict response to cardiac resynchronization therapy in ischemic heart failure patients. $J \mathrm{Nucl}$ Med 2006;47:1565-1570.

25. Henneman MM, Van Der Wall EE, Ypenburg C, et al: Nuclear imaging in cardiac resynchronization therapy. J Nucl Med 2007;48:2001-2010.

26. Chalil S, Foley PWX, Muyhaldeen SA, et al: Late gadolinium enhancement-cardiovascular magnetic resonance as a predictor of response to cardiac resynchronization therapy in patients with ischaemic cardiomyopathy. Europace 2007;9:1031-1037.

27. Ypenburg C, Sieders A, Bleeker GB, et al: Myocardial contractile reserve predicts improvement in left ventricular function after cardiac resynchronization therapy. Am Heart $J$ 2007;154:1160-1165.

28. Lim P, Bars C, Mitchell-Hegg L, et al: Importance of contractile reserve for CRT. Europace 2007;9:739 743.

29. Ypenburg C, Schalij MJ, Bleeker GB, et al: Impact of viability and scar tissue on response to cardiac resynchronization therapy in ischaemic heart failure patients. Eur Heart J 2007;28:33-41.

30. Hummel JP, Lindner JR, Belcik JT, et al: Extent of myocardial viability predicts response to biventricular pacing in ischemic cardiomyopathy. Heart Rhythm 2005;2:1211-1217. 


\section{SÉNÉCHAL, ET AL.}

31. Da Costa A, Thévenin J, Roche F, et al: Prospective validation of stress echocardiography as an identifier of cardiac resynchronization therapy responders. Heart Rhythm 2006;3:406-413.

32. Bleeker GB, Kaandorp TAM, Lamb JH, et al: Effect of posterolateral scar tissue on clinical and echocardiographic improvement after cardiac resynchronization therapy. Circulation 2006;113:969-976.

33. Zoghbi WA, Enriquez-Sarano M, Foster E, et al: Recommendations for evaluation of the severity of native valvular regurgitation with two-dimensional and Doppler echocardiography. J Am Soc Echocardiogr 2003;16:777-802.

34. Bader H, Garrigue S, Lafitte S, et al: Intra-left ventricular electromechanical asynchrony: A new independent predictor of severe cardiac events in heart failure patients. J Am Coll Cardiol 2004;43:248-256.

35. deFilippi CR, Willett DL, Irani WN, et al: Comparison of myocardial contrast echocardiography and lowdose dobutamine stress echocardiography in predicting recovery of left ventricular function after coronary revascularization in chronic ischemic heart disease. Circulation 1995;92:2863-2868.

36. Cigarroa CG, deFilippi CR, Brickner ME, et al: Dobutamine stress echocardiography identifies hibernating myocardium and predicts recovery of left ventricular function after coronary revascularization. Circulation 1993;88:430-436.

37. Schiller NB, Shah PM, Crawford M, et al: Recommendations for quantitation of the left ventricle by twodimensional echocardiography. J Am Soc Echocardiogr 1989;2:358-367.

38. Butter C, Auricchio A, Stellbrink C, et al: Effect of resynchronization therapy stimulation site on the systolic function of heart failure patients. Circulation 2001;104:3026-3029.
39. Qureshi U, Nagueh SF, Afridi I, et al: Dobutamine echocardiography and quantitative restredistribution ${ }^{201}$ TI tomography in myocardial hibernation: Relation of contractile reserve to ${ }^{201}$ TI uptake and comparative prediction of recovery of function. Circulation 1997;95:626-635.

40. Ramahi TM, Longo MD, Cadariu AR, et al: Dobutamine-induced augmentation of left ventricular ejection fraction predicts survival of heart failure patients with severe non-ischemic cardiomyopathy. Eur Heart $J$ 2001;22:849-856.

41. Eichhorn EJ, Grayburn PA, Mayer SA, et al: Myocardial contractile reserve by dobutamine stress echocardiography predicts improvement in ejection fraction with $\beta$-blockade in patients with heart failure. The $\beta$-blocker evaluation of survival trial (BEST). Circulation 2003;108:2336-2341.

42. Cappola TP, Harsch MR, Jessup M, et al: Predictors of remodeling in the CRT Era: Influence of mitral regurgitation, BNP and gender. J Card Fail 2006;12:182188.

43. Cabrera-Bueno F, Garcia-Pinilla JM, PenaHernandez J, et al: Repercussion of functional mitral regurgitation on reverse remodelling in cardiac resynchronization therapy. Europace 2007;9:757-761.

44. Diaz-Infante E, Mont L, Leal J, et al: Predictors of lack of response to resynchronization therapy. Am J Cardiol 2005;95:1436-1440.

45. Reuter S, Garrigue S, Barold SS, et al: Comparison of characteristics in responders versus non-responders with biventricular pacing for drug-resistant congestive heart failure. Am J Cardiol 2002;89:346-350.

46. Gorcsan J, Tanabe M, Bleeker GB, et al: Combined longitudinal and radial dyssynchrony predicts ventricular response after resynchronization therapy. JACC 2007;50:1476-1483. 